\title{
Incorporating Bortezomib in the Management of Plasmablastic Lymphoma
}

\author{
ONYEDIKA UMEANAETO ${ }^{1}$, JOSEPH GAMBOA ${ }^{2}$, JESUS DIAZ ${ }^{2}$, M. NAWAR HAKIM ${ }^{3}$, \\ JAVIER CORRAL ${ }^{1}$, ALEXANDER PHILIPOVSKIY $^{1}$ and SUMIT GAUR ${ }^{1}$ \\ ${ }^{1}$ Department of Internal Medicine, TTUHSC-El Paso, Paul L Foster School of Medicine, El Paso, TX, U.S.A.; \\ ${ }^{2}$ Department of Radiology, TTUHSC-El Paso, Paul L Foster School of Medicine, El Paso, TX, U.S.A.; \\ ${ }^{3}$ Department of Pathology, TTUHSC-El Paso, Paul L Foster School of Medicine, El Paso, TX, U.S.A.
}

\begin{abstract}
Incorporating bortezomib and/or lenalidomide in the management of plasmablastic lymphoma is an attractive option due to the reported high response rates. However, concerns about overlapping toxicities can deter clinicians from incorporating these novel agents into chemotherapy. In this case report we describe a patient with plasmablastic lymphoma, who received both lenalidomide and bortezomib as part of upfront treatment for a high-risk plasmablastic lymphoma. After completing intensive chemotherapy, the patient was transitioned to a regimen of daily lenalidomide and biweekly bortezomib to decrease the chance of relapse. This maintenance phase was given for 6 months and was well tolerated. Despite having multiple adverse risk factors, the patient remains in remission, 18 months following diagnosis.
\end{abstract}

Plasmablastic lymphoma (PBL) is a distinct subtype of diffuse large B cell lymphoma (DLBCL), in which the malignant cells resemble plasmablasts expressing plasma cell markers (CD38, CD 138, MUM-1). Although initially described in HIV-infected patients, it is now known to affect both immunocompromised and immunocompetent patients. It is an aggressive disease with a poor prognosis. Although initially described more than 20 years ago, no prospective trials have been completed in this subtype of DLBCL reflecting the rarity of the disease. As such, there is no uniform standard of care. Management decisions are guided by case series and expert opinions. There is a uniform consensus that cyclophosphamide-, doxorubicin- vincristine-

Correspondence to: Sumit Gaur, MD, Associate Professor, Hematology and Medical Oncology, TTUHSC-El Paso, 4800 Alberta Avenue, El Paso, TX 79905, U.S.A. Tel: +1 915215 5200, Fax: +1 9152158640, e-mail: sumit.gaur@ttuhsc.edu

Key Words: Plasmablastic lymphoma, bortezomib, lenalidomide, $\mathrm{EPOCH}$, maintenance therapy. and prednisone (CHOP)-like regimens are inadequate. The disease bears resemblance to multiple myeloma both in its pathological and clinical features. Thus, agents used in multiple myeloma have been tried and have shown activity. However, how to best incorporate these in the management of this disease is not clear.

Herein, we describe a patient with stage IV, IPI (international prognostic index) score 3 (high intermediate risk), HIV negative plasmablastic lymphoma who had a less-thancomplete response on a PET (positron emission tomography) scan at the end of 6 cycles of dose adjusted EPOCH (etoposide, prednisone, vincristine, cyclophosphamide, doxorubicin). She was then transitioned to daily lenalidomide and bortezomib every 2 weeks because she declined a stem cell transplant. "Maintenance phase" with lenalidomide and bortezomib was continued for 6 months. She has been in remission for 18 months since initial diagnosis.

\section{Case Report}

A 55-year-old female presented with a rapidly enlarging mass in her mouth, diplopia, back pain, subjective fever and a 15-pound weight loss over 1 month. She had initially noticed the mass in her mouth 1 month ago and treated it with topical anesthetic. The mass had continued to enlarge and started bleeding 1 day before her emergency room visit. One week before presentation, she started having diplopia. She reported drenching night sweats for one month along with headaches and back pain. She had a past medical history of type II diabetes mellitus for which she took metformin and hypertension controlled with Lisinopril. She was married, in a monogamous relationship and family history was unremarkable. On exam, she looked pale, cachectic and had a resting pulse rate of $106 /$ min with normal temperature and blood pressure. Exophthalmos was noted on the right eye. A $5 \times 7 \mathrm{~cm}$ ulcerated, bleeding lesion was present over the left hard palate. Liver and spleen were not palpable and there was no lymphadenopathy. Muscle 
strength, gait and sensations were intact. Laboratory data showed a hemoglobin of $6.4 \mathrm{gm} / \mathrm{dl}$ (12-15) with normal white blood cell (WBC) and platelet counts. Serum calcium was $12.4 \mathrm{mg} / \mathrm{dl}$ (8.4-10.2), blood urea nitrogen (BUN) was $18 \mathrm{mg} / \mathrm{dl}$ and creatinine was $1.2 \mathrm{mg} / \mathrm{dl}$ with an estimated glomerular filtration rate of $47 \mathrm{ml} / \mathrm{min}$. LDH (lactate dehydrogenase) was $225 \mathrm{IU} / 1$ (120-246). Serology for HIV and hepatitis viruses was negative. Cerebrospinal fluid (CSF) analysis showed only $1 \mathrm{WBC} / \mathrm{microl}$, normal protein and CSF cytology did not reveal malignant cells.

Computed tomography (CT) showed innumerable osteolytic lesions in the calvarium, skull base, and cervical, thoracic and lumbar spine with enhancing soft tissue masses in the skull, bilateral maxillary sinuses, right orbit and mandible (Figure 1a). MRI confirmed above findings; some of the soft tissue masses in the calvarium were infiltrating into the underlying Dura mater (Figure 1b).

Biopsy of the left palatine mass showed a kappa restricted, plasmablastic lymphoma. Tumor cells were positive for CD138, CD79a, CD56 and epithelial membrane antigen (EMA). CD20 was not expressed (Figure 2). In situ hybridization, showed the tumor cell to be positive for Epstein-Barr virus.

The patient was emergently started on infusional doseadjusted EPOCH (etoposide, prednisone, vincristine, cyclophosphamide, doxorubicin). She was successfully elevated to level 2 dosing from 3 rd cycle onwards. She received intra-thecal treatments with methotrexate with each cycle. A PET scan was obtained at the end of the 6 cycles of therapy, which showed persistent activity in left mandibular ramus, right shoulder, right clavicle, right proximal femur and bilateral iliac bones. In addition, increased activity was seen diffusely over the spine. Standard uptake values (SUV) in these areas ranged from 3-6 (Figure 1c).

She was advised about the high risk of symptomatic relapse and high dose chemotherapy with autologous stem cell transplant was recommended. She declined and was subsequently placed on lenalidomide $10 \mathrm{mg}$ daily and bortezomib $1.3 \mathrm{mg} / \mathrm{m}^{2}$ every 2 weeks. Treatment was very well tolerated with no complications. She received this regimen for 6 months and has been followed clinically since then. At last follow up, 18 months from initial diagnosis, she has not had a relapse.

\section{Discussion}

Patients with advanced plasmablastic lymphoma have a poor prognosis. A review of 76 cases of HIV-negative plasmablastic lymphoma found a median survival of 9 months and a 2-year survival rate of $10 \%$ (1). Morscio et al. performed a metaanalysis of 300 cases of plasmablastic lymphoma and reported a median survival of 11 months in HIV-negative immunocompetent cases (2). In an attempt to improve
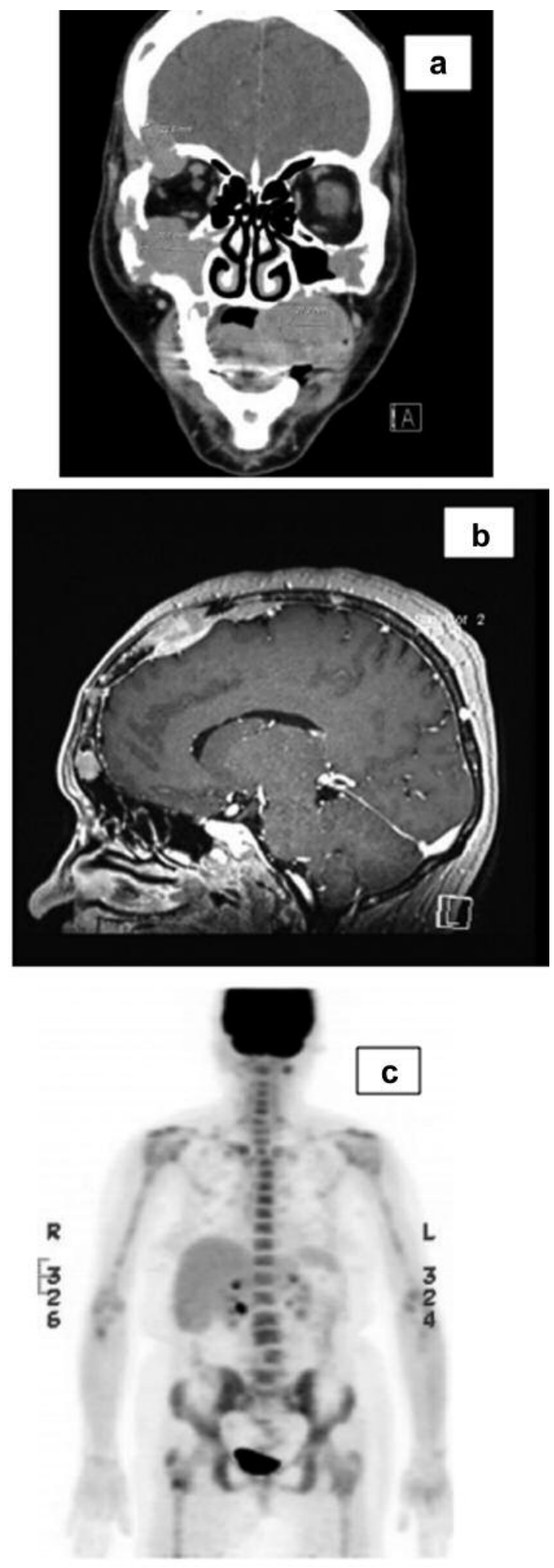

Figure 1. Radiology images. CT (A) and MRI (B) showing soft tissue masses in left and right maxillary area, right orbit and calvarium with infiltration of underlying Dura Mater. (C) PET scan at the end of 6 cycles of EPOCH showing residual activity in left mandible, right shoulder, right proximal femur, both iliac bones and in the spine. 

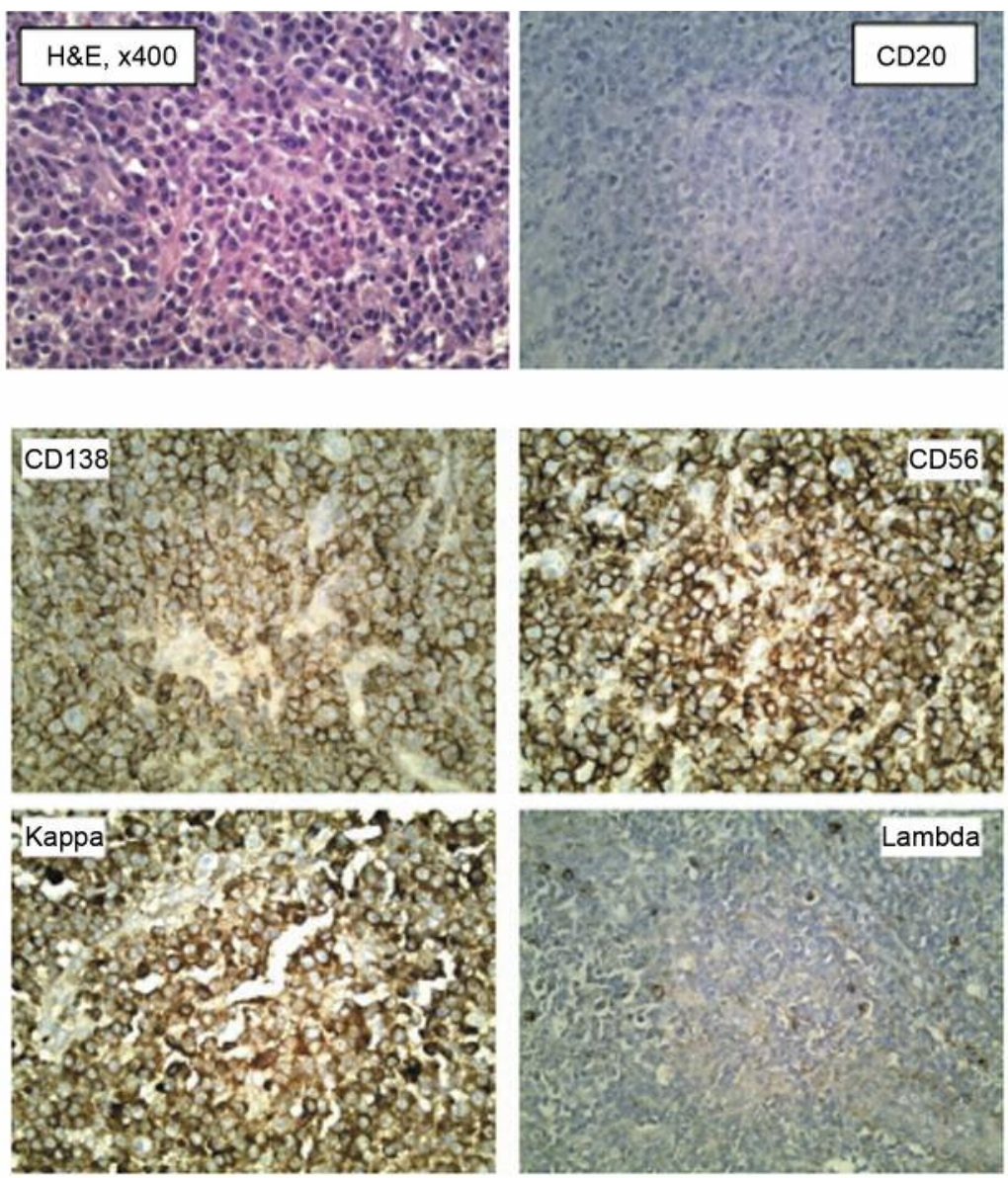

Figure 2. Pathology images. The tumor cells display plasmablastic appearance with eccentric nuclei, moderate amount of amphiphilic cytoplasm and some cells with prominent large nucleoli. The tumor cells are positive for CD138, CD56 and show Kappa light chains restriction pattern.

outcomes, current guidelines recommend more aggressive regimens than traditional $\mathrm{CHOP}(3)$. Amongst these infusional dose-adjusted regimens, EPOCH is the most commonly used. Similar attempts have been made to decrease the rate of relapse by performing autologous stem cell transplantation in patients who seem to be responding to chemotherapy. However, in the absence of any direct head-to-head comparison to CHOP, it is unclear if these more intense regimens improve survival.

Given the significant overlap in pathological and clinical features of multiple myeloma and plasmablastic lymphoma, there has been interest in incorporating novel agents with activity in multiple myeloma, in the treatment of plasmablastic lymphoma. Of these, bortezomib has been thought to be the most promising. The combination of bortezomib with EPOCH regimen was first described in 2015 by Castillo et al. In this original case series, all three treated patients achieved a durable remission (4). A 2017 PubMed-based literature review of cases found that when combined with chemotherapy, the complete response rate to bortezomib based chemotherapy regimen is $89 \%$ with a 2 year survival of $55 \%$. In the relapsed setting, bortezomib based combination chemotherapy induced a complete response in $16 \%$ with 2-year survival of $27 \%$ (5). More recently, Castillo et al. retrospectively reviewed outcomes of 16 patients with plasmablastic lymphoma treated with bortezomib plus EPOCH regimen and reported a complete response rate of $94 \%$ and 5-year survival rate of $65 \%$ (6).

Despite these encouraging results, it is unclear as to how best to incorporate bortezomib in the treatment of plasmablastic lymphomas. Concurrent use of bortezomib with chemotherapy is toxic with high rates of neuropathy, infections and febrile neutropenia (5). In the original case series of three patients described by Castillo et al., two developed grade 4 thrombocytopenia, one had reactivation of herpes zoster and one developed small bowel obstruction, prompting discontinuation of vincristine (4). Dose adjustment of doxorubicin, etoposide and cyclophosphamide, based on nadir neutrophil and platelet counts in the preceding 
chemotherapy cycle, is a key component of the da-EPOCH regimen. Dose of vincristine requires adjustment based on the degree of neuropathy (7). Neuropathy is common with bortezomib and both bortezomib and lenalidomide cause cytopenia. Therefore, using them with EPOCH can potentially compromise the possibility of dose escalation.

In this case report we describe an alternative way of incorporating bortezomib, in the upfront management of a patient with plasmablastic lymphoma. Delaying the introduction of bortezomib and lenalidomide in the treatment regimen till all planned cycles of EPOCH were completed allowed for successful dose escalation of chemotherapy required for pharmacodynamics, the clarification of the doseeffect relationship.

A "maintenance strategy" of using less intensive therapy to prevent relapses after completing intensive therapy is commonly used in many hematologic malignancies. For example, a regimen containing daily mercaptopurine with weekly pulses of methotrexate and monthly pulses of prednisone and vincristine is recommended to patients with acute lymphoblastic leukemia after completing intensive therapy (8). Maintenance therapy with bortezomib and lenalidomide have been shown to delay relapse and is commonly offered to patients with multiple myeloma $(9,10)$. Maintenance therapy with lenalidomide has also been shown to improve survival in elderly patients with diffuse large B cell lymphoma (11).

We used a bortezomib, lenalidomide-based maintenance regimen in our patient because her high IPI score and positive PET scan at the end of therapy predicted a high risk for relapse and she declined an autologous stem cell transplant (12). Prolonged exposure to lenalidomide in patients with multiple myeloma has been associated with risk of secondary malignancies. Bortezomib can cause cumulative neuropathy. Given the high proliferative rate of plasmablastic lymphoma, most of the relapses are expected to occur during or shortly after completing therapy. Therefore, we elected to continue maintenance therapy for 6 months and not longer.

In conclusion, a time-limited regimen of daily lenalidomide and biweekly bortezomib following completion of dose adjusted EPOCH allowed for the incorporation of these two agents safely in the successful management of a patient with plasmablastic lymphoma. Larger clinical trials are needed to fully evaluate the efficacy of this approach. In the meantime, clinicians who are concerned about overlapping toxicities when using chemotherapy concurrently with bortezomib can consider this approach as an option for their patients with plasmablastic lymphoma.

\section{Conflicts of Interest}

The Authors have no conflicts of interest to declare regarding this study.

\section{Authors' Contributions}

Onyedika Umeanaeto: Identified the case, gathered data and wrote first draft; Joseph Gamboa and Jesus Diaz: Reviewed radiology images; M Nawar Hakim: Reviewed pathology slides; Javier Corral, Alexander Philopovskiy and Sumit Gaur: Performed literature review and assisted with finalizing the manuscript.

\section{References}

1 Castillo JJ, Winer ES, Stachurski D, Perez K, Jabbour M, Milani C, Colvin GA and Butera JN: HIV-negative plasmablastic lymphoma: not in the mouth. Clin Lymphoma Myeloma Leuk 11(2): 185-189, 2011. PMID: 21575922. DOI: 10.1016/j.clml. 2011.03.008

2 Morscio J, Dierickx D, Nijs J, Verhoef G, Bittoun E, Vanoeteren X, Wlodarska I, Sagaert X and Tousseyn T: Clinicopathologic comparison of plasmablastic lymphoma in HIV-positive, immunocompetent, and posttransplant patients: single-center series of 25 cases and meta-analysis of 277 reported cases. Am J Surg Pathol 38(7): 875-886, 2014. PMID: 24832164. DOI: 10.1097/PAS.0000000000000234

3 National comprehensive cancer network guidelines. B-Cell Lymphomas. (Version 4.2019). Available at: https://www. nccn.org/professionals/physician_gls/pdf/b-cell.pdf

4 Castillo JJ, Reagan JL, Sikov W and Winer ES: Bortezomib in combination with infusional dose-adjusted EPOCH for the treatment of plasmablastic lymphoma. Br J Haematol 169: 352355, 2015. PMID 25612847. DOI: 10.1111/bjh.1330

5 Guerrero-Garcia TA, Mogollon RJ and Castillo JJ: Bortezomib in plasmablastic lymphoma: a glimpse of hope in a hard to treat disease. Leuk Res 62: 12-16, 2017. PMID: 2896390. DOI: 10.1016/j.leukres.2017.09.020

6 Castillo JJ, Guerrero-Garcia T, Baldini F, Tchernonog E, Cartron G, Ninkovic S, Cwynarski K, Dierickx D, Tousseyn T, Lansigan F, Linnik Y, Mogollon R, Navarro JT, Olszewski AJ, Reagan JL, Fedele P, Gilbertson M, Grigoriadis G and Bibas M: Bortezomib plus EPOCH is effective as frontline treatment in patients with plasmablastic lymphoma. Br J Haematol 184: 679-682, 2019. PMID: 29527667. DOI: 10.1111/bjh.15156

7 Wilson WH, Grossbard ML, Pittaluga S, Cole D, Pearson D, Drbohlav N, Steinberg SM, Little RF, Janik J, Gutierrez M, Raffeld M, Staudt L, Cheson BD, Longo DL, Harris N, Jaffe ES, Chabner BA, Wittes R and Balis F: Dose adjusted EPOCH chemotherapy for untreated large $B$ cell lymphoma: a pharmacodynamic approach with high efficacy. Blood 99: 26852693, 2002. PMID: 11929754. DOI: 10.1182/blood.v99.8.2685

8 National comprehensive cancer network guidelines. Acute lymphoblastic leukemia. (Version 2.2019). Available at: https://www.nccn.org/professionals/physician_gls/pdf/all.pdf

9 Mccarthy PL, Holstein SA, Petrucci MT, Richardson PG, Hulin C, Tosi P, Bringhen S, Musto P, Anderson KC, Caillot D, Gay F, Moreau P, Marit G, Jung SH, Yu Z, Winograd B, Knight RD, Palumbo A and Attal M: Lenalidomide maintenance after autologous stem cell transplantation in newly diagnosed multiple myeloma: A meta-analysis. J Clin Oncol 10: 3279-3289, 2017. PMID: 28742454. DOI:10.1200/JCO.2017.72.6679

10 Sonneveld P, Schmidt-Wolf IG, van-der-Holt B, El Jarari L, Bertsch U, Salwender H, Zweegman S, Vellenga E, Broyl A, 
Blau IW, Weisel KC, Wittebol S, Bos GM, Stevens-Kroef M, Scheid C, Pfreundschuh M, Hose D, Jauch A, van der Velde H, Raymakers R, Schaafsma MR, Kersten MJ, van Marwijk-Kooy M, Duehrsen U, Lindemann W, Wijermans PW, Lokhorst HM and Goldschmidt HM: Bortezomib induction and maintenance treatment in patients with newly diagnosed multiple myeloma: results of the randomized phase III HOVON 65/GMMG-HD4 trial. J Clin Oncol 20: 2946-2955, 2012. PMID: 22802322. DOI: 10.1200/JCO.2011.39.6820

11 Thiemblemont C, Tilly H, Gomes da Silva M, Casasnovas RO, Fruchart C, Morschhauser F, Haioun C, Lazarovici J, Grosicka A, Perrot A, Trotman J, Sebban C, Caballero D, Greil R, van Eygen K, Cohen AM, Gonzalez H, Bouabdallah R, Oberic L, Corront B, Choufi B, Lopez-Guillermo A, Catalano J, Van Hoof A, Briere J, Cabeçadas J, Salles G, Gaulard P, Bosly A and Coiffier B: Lenalidomide maintenance compared with placebo in responding elderly patients with diffuse large $\mathrm{b}$ cell lymphoma treated with first line rituximab plus cyclophosphamide, doxorubicin, vincristine and prednisone. J Clin Oncol 35: 24732481, 2017. PMID: 28426350. DOI: 10.1200/JCO.2017.72.6984

12 Al Tabba Y, Tchemonog E, Faurie P, Cottereau AS, Monjanel H, Bonnet A, Le Gouill S, Gyan E, Ghesquieres H, MarianoGoulart D and Cartron G: Post-treatment positron emission tomography is highly predictive of outcome in plasmablastic lymphoma. Eur J Nucl Med Imaging 10: 1705-1709, 2018. PMID: 29679112. DOI: 10.1007/s00259-018-4020-5
Received June 15, 2019

Revised July 1, 2019

Accepted July 8, 2019 